\title{
Time-dependent one-dimensional electromagnetic wave propagation in inhomogeneous media: exact solution in terms of transmutations and Neumann series of Bessel functions
}

\author{
Kira V. Khmelnytskaya ${ }^{1}$, Vladislav V. Kravchenko ${ }^{2}$, Sergii M. Torba ${ }^{2 *}$ \\ ${ }^{1}$ Faculty of Engineering, Autonomous University of Queretaro, \\ Cerro de las Campanas s/n, Querétaro, Qro., CP 76010, México \\ ${ }^{2}$ Department of Mathematics, Cinvestav, Campus Querétaro \\ Libramiento Norponiente \#2000, Querétaro, Qro., CP 76230, México \\ khmel@uaq.edu.mx, vkravchenko@math.cinvestav.edu.mx, storba@math.cinvestav.edu.mx
}

November 1, 2019

\begin{abstract}
The time-dependent Maxwell system describing electromagnetic wave propagation in inhomogeneous isotropic media in the one-dimensional case reduces to a Vekua-type equation for bicomplex-valued functions of a hyperbolic variable, see [7. In [5] using this reduction a representation of a general solution of the system was obtained in terms of a couple of Darbouxassociated transmutation operators [8]. In [6] a Fourier-Legendre expansion of transmutation integral kernels was obtained. This expansion is used in the present work for obtaining an exact solution of the problem of the transmission of a normally incident electromagnetic timedependent plane wave through an arbitrary inhomogeneous layer. The result can be used for efficient computation of the transmitted modulated signals. In particular, it is shown that in the classical situation of a signal represented in terms of a trigonometric Fourier series the solution of the problem can be written in the form of Neumann series of Bessel functions with exact formulas for the coefficients. The representation lends itself to numerical computation.
\end{abstract}

\section{Introduction}

In the present work, the 1+1 Maxwell system for isotropic inhomogeneous media is considered. In [5] a representation for its general solution was obtained in terms of a couple of Darboux-associated transmutation (transformation) integral operators (for a recent overview of transmutation operator theory and applications we refer to [4] and [14]). Here we develop further this representation applying a recent result from [6] where a Fourier-Legendre expansion of the transmutation integral kernels was obtained with explicit formulas for the coefficients. The main result of the present work is an exact solution of the classical problem of time-dependent plane electromagnetic wave propagation through an inhomogeneous medium with an arbitrary profile. The solution has a form of a series, the coefficients of which are computed recursively. In particular, if the incoming signal is represented in terms of a Fourier series the solution of the problem acquires the form of a Neumann series of Bessel functions. An important feature of this solution representation consists in the fact that its truncated version approximates the exact solution equally well for small and for large values

${ }^{*}$ The authors acknowledge the support from CONACYT, Mexico via the projects 222478 and 284470. 
of the frequency which means that even when the partial sums of the Fourier series contain a large number of members and independently of the largeness of the carrier frequency the approximation of the solution presented here does not deteriorate.

\section{The 1+1 Maxwell system and the hyperbolic Vekua equation}

The Maxwell system for an isotropic inhomogeneous sourceless medium has the form

$$
\begin{aligned}
\operatorname{div}(\mu \mathbf{H}) & =0, & & \operatorname{rot} \mathbf{H}=\varepsilon \partial_{t} \mathbf{E}, \\
\operatorname{div}(\varepsilon \mathbf{E}) & =0, & & \operatorname{rot} \mathbf{E}=-\mu \partial_{t} \mathbf{H},
\end{aligned}
$$

where $\varepsilon$ and $\mu$ are real-valued functions of spatial coordinates, $\mathbf{E}$ and $\mathbf{H}$ are real-valued vector fields depending on $t$ and on spatial variables. In the case when all the magnitudes involved are independent of two spatial coordinates, say, $x_{2}$ and $x_{3}, \varepsilon=\varepsilon\left(x_{1}\right)$ and $\mu=$ Const, the resulting $1+1$ Maxwell system for an inhomogeneous medium can be written in the form

$$
\varepsilon(x) \partial_{t} \mathcal{E}=i \partial_{x} \mathcal{H}, \quad i \partial_{x} \mathcal{E}=-\mu \partial_{t} \mathcal{H}
$$

where $\mathcal{E}=E_{2}+i E_{3}, \mathcal{H}=H_{2}+i H_{3}, x=x_{1}$. Denote $c(x)=1 / \sqrt{\varepsilon(x) \mu}$. It is assumed nonvanishing.

In [7] it was shown that system (1) can be transformed into the following Vekua-type hyperbolic equation

$$
\partial_{\bar{z}} W-\frac{f^{\prime}}{2 f} \bar{W}=0
$$

where $\partial_{\bar{z}}=\frac{1}{2}\left(\partial_{\xi}-j \partial_{t}\right), j$ is a hyperbolic imaginary unit, $j^{2}=1$ commuting with $i, W$ is a bicomplex-valued function of the real variables $\xi$ and $t, W=u+v j$ and $u, v$ are complex valued (containing the imaginary unit $i$ ). The function $f$ is real valued and depends on $\xi$ only. The conjugation with respect to $j$ is denoted by the bar, $\bar{W}=u-v j$.

The relation between (1) and (2) involves the change of the independent variable $\xi(x)=$ $\sqrt{\mu} \int_{0}^{x} \sqrt{\varepsilon(s)} d s$. The function $f$ in $(2)$ is related to $\varepsilon$ and $\mu$ by the equality $f(\xi)=\sqrt{\widetilde{c}(0)} / \sqrt{\widetilde{c}(\xi)}$ where and below the tilde means that a function of $x$ is written as a function of $\xi, \widetilde{c}(\xi(x))=c(x)$. The bicomplex-valued function $W$ is written in terms of $E$ and $H$ as follows

$$
W(\xi, t)=\sqrt{\widetilde{c}(\xi)}(\sqrt{\widetilde{\varepsilon}(\xi)} \widetilde{\mathcal{E}}(\xi, t)+i j \sqrt{\mu} \widetilde{\mathcal{H}}(\xi, t)) .
$$

For the scalar components of a bicomplex-valued function $w=u+v j$ the following notations will be used

$$
\mathcal{R}(w)=u=\frac{1}{2}(w+\bar{w}), \quad \mathcal{I}(w)=v=\frac{1}{2 j}(w-\bar{w})
$$

and

$$
w^{ \pm}:=\mathcal{R}(w) \pm \mathcal{I}(w)
$$

Then from (3) we have

$$
\widetilde{\mathcal{E}}(\xi, t)=\frac{1}{\sqrt{\widetilde{c}(\xi) \widetilde{\varepsilon}(\xi)}} \mathcal{R}(W(\xi, t))
$$

and

$$
\widetilde{\mathcal{H}}(\xi, t)=-\frac{i}{\sqrt{\widetilde{c}(\xi) \mu}} \mathcal{I}(W(\xi, t))
$$




\section{A general solution of the hyperbolic Vekua equation}

Together with the Vekua equation (2) consider its special case, the hyperbolic Cauchy-Riemann system

$$
\partial_{\bar{z}} w=0
$$

which was studied in several publications (see, e.g., [10], [11, [16] and more recent [9]). Its general solution can be written in the form

$$
w(\xi, t)=P^{+} \Phi(t+\xi)+P^{-} \Psi(t-\xi)
$$

where $\Phi$ and $\Psi$ are arbitrary continuously differentiable scalar functions (complex valued containing the imaginary unit $i$ ) and $P^{ \pm}=\frac{1}{2}(1 \pm j)$.

In [9] there was established a relation between solutions of (7) and (2). Any solution of (2) can be represented in the form

$$
W(\xi, t)=T_{f}[\mathcal{R}(w(\xi, t))]+j T_{1 / f}[\mathcal{I}(w(\xi, t))]
$$

where $w$ is a solution of $(7), T_{f}$ and $T_{1 / f}$ are Darboux-associated transmutation operators defined in [8], see also [2] and [9]. The operators $T_{f}$ and $T_{1 / f}$ are applied with respect to the variable $\xi$ and have the form of second-kind Volterra integral operators,

$$
T_{f} u(\xi)=u(\xi)+\int_{-\xi}^{\xi} \mathbf{K}_{f}(\xi, \tau) u(\tau) d \tau
$$

and

$$
T_{1 / f} u(\xi)=u(\xi)+\int_{-\xi}^{\xi} \mathbf{K}_{1 / f}(\xi, \tau) u(\tau) d \tau
$$

with continuously differentiable kernels $\mathbf{K}_{f}$ and $\mathbf{K}_{1 / f}$.

In [6] a representation of the transmutation kernels in the form of Fourier-Legendre series was obtained. Namely, the transmutation kernels $\mathbf{K}_{f}$ and $\mathbf{K}_{1 / f}$ have the form

$$
\mathbf{K}_{f}(\xi, \tau)=\sum_{n=0}^{\infty} \frac{a_{n}(\xi)}{\xi} P_{n}\left(\frac{\tau}{\xi}\right)
$$

and

$$
\mathbf{K}_{1 / f}(\xi, \tau)=\sum_{n=0}^{\infty} \frac{b_{n}(\xi)}{\xi} P_{n}\left(\frac{\tau}{\xi}\right)
$$

where $P_{n}$ stands for the Legendre polynomial of order $n$, for every $\xi>0$ the series converge uniformly with respect to $\tau \in[-\xi, \xi]$, and for the coefficients $a_{n}$ and $b_{n}, n=0,1,2, \ldots$ explicit formulas are obtained. In order to write them down we introduce the systems of functions $\left\{\varphi_{k}\right\}_{k=0}^{\infty}$ and $\left\{\psi_{k}\right\}_{k=0}^{\infty}$ defined as follows.

Consider two sequences of recursive integrals

$$
X^{(0)} \equiv 1, \quad X^{(n)}(\xi)=n \int_{0}^{\xi} X^{(n-1)}(s)\left(f^{2}(s)\right)^{(-1)^{n}} \mathrm{~d} s, \quad n=1,2, \ldots
$$

and

$$
\widetilde{X}^{(0)} \equiv 1, \quad \widetilde{X}^{(n)}(\xi)=n \int_{0}^{\xi} \widetilde{X}^{(n-1)}(s)\left(f^{2}(s)\right)^{(-1)^{n-1}} \mathrm{~d} s, \quad n=1,2, \ldots
$$


The two families of functions $\left\{\varphi_{k}\right\}_{k=0}^{\infty}$ and $\left\{\psi_{k}\right\}_{k=0}^{\infty}$ are constructed according to the rules

$$
\varphi_{k}(\xi)= \begin{cases}f(\xi) X^{(k)}(\xi), & k \text { odd } \\ f(\xi) \widetilde{X}^{(k)}(\xi), & k \text { even }\end{cases}
$$

and

$$
\psi_{k}(\xi)= \begin{cases}\frac{\widetilde{X}^{(k)}(\xi)}{f(\xi)}, & k \text { odd }, \\ \frac{X^{(k)}(\xi)}{f(\xi)}, & k \text { even. }\end{cases}
$$

The coefficients $a_{n}$ and $b_{n}$ in (9) and (10) admit the following representation [6]

$$
a_{n}(\xi)=\frac{2 n+1}{2}\left(\sum_{k=0}^{n} \frac{l_{k, n} \varphi_{k}(\xi)}{\xi^{k}}-1\right)
$$

and

$$
b_{n}(\xi)=\frac{2 n+1}{2}\left(\sum_{k=0}^{n} \frac{l_{k, n} \psi_{k}(\xi)}{\xi^{k}}-1\right)
$$

where $l_{k, n}$ is $k$-th power's coefficient of the Legendre polynomial of order $n$, that is $P_{n}(x)=$ $\sum_{k=0}^{n} l_{k, n} x^{k}$.

Besides these direct formulas for the coefficients $a_{n}$ and $b_{n}$, in [6] a recurrent integration procedure for their computation was proposed, convenient for numerical applications.

Substitution of (9) and (10) into (8) leads to the following representation of a general solution of (2)

$$
W(\xi, t)=w(\xi, t)+\sum_{n=0}^{\infty} \frac{a_{n}(\xi)}{\xi} \int_{-\xi}^{\xi} P_{n}\left(\frac{\tau}{\xi}\right) \mathcal{R}(w(\tau, t)) \mathrm{d} \tau+j \sum_{n=0}^{\infty} \frac{b_{n}(\xi)}{\xi} \int_{-\xi}^{\xi} P_{n}\left(\frac{\tau}{\xi}\right) \mathcal{I}(w(\tau, t)) \mathrm{d} \tau
$$

where $w$ is a general solution of (7).

\section{Normally incident plane wave propagation through an inhomo- geneous medium}

In this section we study the classical problem of a normally incident plane wave propagation through an inhomogeneous medium (see, e.g., [12, Chapter 8]). The electromagnetic field $\mathcal{E}$ and $\mathcal{H}$ satisfying (1) is supposed to be known at $x=0$,

$$
\mathcal{E}(0, t)=\mathcal{E}_{0}(t) \quad \text { and } \quad \mathcal{H}(0, t)=\mathcal{H}_{0}(t), \quad t \in[\alpha, \beta] .
$$

We assume $\mathcal{E}_{0}$ and $\mathcal{H}_{0}$ to be continuously differentiable functions.

Problem (1), (15) can be reformulated in terms of the function (3). Find a solution of (2) satisfying the condition

$$
W(0, t)=W_{0}(t)
$$

where

$$
W_{0}=\sqrt{c(0) \varepsilon(0)} \mathcal{E}_{0}+i j \sqrt{c(0) \mu} \mathcal{H}_{0}
$$

is a given continuously differentiable function. Then the following statement is valid. 
Theorem 1 The unique solution of Problem (2), (16) has the form

$$
\begin{aligned}
W(\xi, t)= & \frac{1}{2}\left(W_{0}^{+}(t+\xi)+W_{0}^{-}(t-\xi)\right)+\frac{1}{2} \sum_{n=0}^{\infty} \frac{a_{n}(\xi)}{\xi} \int_{-\xi}^{\xi} P_{n}\left(\frac{\tau}{\xi}\right)\left(W_{0}^{+}(t+\tau)+W_{0}^{-}(t-\tau)\right) \mathrm{d} \tau \\
& +\frac{j}{2}\left(W_{0}^{+}(t+\xi)-W_{0}^{-}(t-\xi)\right)+\frac{j}{2} \sum_{n=0}^{\infty} \frac{b_{n}(\xi)}{\xi} \int_{-\xi}^{\xi} P_{n}\left(\frac{\tau}{\xi}\right)\left(W_{0}^{+}(t+\tau)-W_{0}^{-}(t-\tau)\right) \mathrm{d} \tau \\
= & P^{+} W_{0}^{+}(t+\xi)+P^{-} W_{0}^{-}(t-\xi)+\frac{1}{2 \xi} \sum_{n=0}^{\infty}\left(a_{n}(\xi)+j b_{n}(\xi)\right) \int_{-\xi}^{\xi} P_{n}\left(\frac{\tau}{\xi}\right) W_{0}^{+}(t+\tau) \mathrm{d} \tau \\
& +\frac{1}{2 \xi} \sum_{n=0}^{\infty}\left(a_{n}(\xi)-j b_{n}(\xi)\right) \int_{-\xi}^{\xi} P_{n}\left(\frac{\tau}{\xi}\right) W_{0}^{-}(t-\tau) \mathrm{d} \tau
\end{aligned}
$$

from which the unique solution of Problem (1), (15) is obtained by means of (5), (6) and an inverse change of the variable $\xi \rightarrow x$.

Proof. The unique solution of Problem (2), 16) has the form [5]

$$
W(\xi, t)=\frac{1}{2} T_{f}\left[W_{0}^{+}(t+\xi)+W_{0}^{-}(t-\xi)\right]+\frac{j}{2} T_{1 / f}\left[W_{0}^{+}(t+\xi)-W_{0}^{-}(t-\xi)\right] .
$$

Due to $(9)$ and $(10)$ this can be written in the form $(18)$.

For some practically interesting initial data the integrals in (18) can be evaluated in a closed form. Let us consider one such example corresponding to modulated electromagnetic waves which are represented as partial sums of Fourier series

$$
\mathcal{E}_{0}(t)=\sum_{m=-M}^{M} \alpha_{m} e^{i\left(\omega_{0}+m \omega\right) t} \quad \text { and } \quad \mathcal{H}_{0}(t)=\sum_{m=-M}^{M} \beta_{m} e^{i\left(\omega_{0}+m \omega\right) t} .
$$

This leads to a similar form for the initial data $W_{0}$ in $(16)$,

$$
W_{0}(t)=\sum_{m=-M}^{M} c_{m} e^{i\left(\omega_{0}+m \omega\right) t}
$$

where the bicomplex numbers $c_{m}$ are related to $\alpha_{m}, \beta_{m} \in \mathbb{C}$ as follows

$$
c_{m}=\sqrt{c(0)}\left(\sqrt{\varepsilon(0)} \alpha_{m}+i j \sqrt{\mu} \beta_{m}\right) .
$$

Note that $W_{0}^{ \pm}(t \pm \xi)=\sum_{m=-M}^{M} c_{m}^{ \pm} e^{i\left(\omega_{0}+m \omega\right)(t \pm \xi)}$ and due to $(18)$, the solution of Problem (2), (16) with the initial data (21) is given by the formula

$$
\begin{aligned}
W(\xi, t)= & P^{+} \sum_{m=-M}^{M} c_{m}^{+} e^{i\left(\omega_{0}+m \omega\right)(t+\xi)}+P^{-} \sum_{m=-M}^{M} c_{m}^{-} e^{i\left(\omega_{0}+m \omega\right)(t-\xi)} \\
& +\frac{1}{2 \xi} \sum_{n=0}^{\infty}\left(a_{n}(\xi)+j b_{n}(\xi)\right) \int_{-\xi}^{\xi} P_{n}\left(\frac{\tau}{\xi}\right) \sum_{m=-M}^{M} c_{m}^{+} e^{i\left(\omega_{0}+m \omega\right)(t+\tau)} \mathrm{d} \tau \\
& +\frac{1}{2 \xi} \sum_{n=0}^{\infty}\left(a_{n}(\xi)-j b_{n}(\xi)\right) \int_{-\xi}^{\xi} P_{n}\left(\frac{\tau}{\xi}\right) \sum_{m=-M}^{M} c_{m}^{-} e^{i\left(\omega_{0}+m \omega\right)(t-\tau)} \mathrm{d} \tau
\end{aligned}
$$


Using formula 2.17.5(1) from [13] the integrals here can be evaluated,

$$
\int_{-\xi}^{\xi} P_{n}\left(\frac{\tau}{\xi}\right) e^{i\left(\omega_{0}+m \omega\right) \tau} \mathrm{d} \tau=2 \xi e^{\frac{n \pi i}{2}} j_{n}\left(\left(\omega_{0}+m \omega\right) \xi\right)
$$

and

$$
\int_{-\xi}^{\xi} P_{n}\left(\frac{\tau}{\xi}\right) e^{-i\left(\omega_{0}+m \omega\right) \tau} \mathrm{d} \tau=(-1)^{n} 2 \xi e^{\frac{n \pi i}{2}} j_{n}\left(\left(\omega_{0}+m \omega\right) \xi\right)
$$

where $j_{n}$ stands for the spherical Bessel function of order $n$. Thus, the bicomplex-valued function $W$ from 22 takes the form

$$
\begin{aligned}
W(\xi, t)= & \sum_{m=-M}^{M} e^{i\left(\omega_{0}+m \omega\right) t}\left(P^{+} c_{m}^{+} e^{i\left(\omega_{0}+m \omega\right) \xi}+P^{-} c_{m}^{-} e^{-i\left(\omega_{0}+m \omega\right) \xi}\right. \\
& +c_{m}^{+} \sum_{n=0}^{\infty}\left(a_{n}(\xi)+j b_{n}(\xi)\right) e^{\frac{n \pi i}{2}} j_{n}\left(\left(\omega_{0}+m \omega\right) \xi\right) \\
& \left.+c_{m}^{-} \sum_{n=0}^{\infty}(-1)^{n}\left(a_{n}(\xi)-j b_{n}(\xi)\right) e^{\frac{n \pi i}{2}} j_{n}\left(\left(\omega_{0}+m \omega\right) \xi\right)\right) .
\end{aligned}
$$

In order to obtain the corresponding formula for the solution of Problem (1), (15) with the initial data of the form (20), we notice that

$$
\begin{aligned}
\mathcal{R}(W(\xi, t))= & \sum_{m=-M}^{M} e^{i\left(\omega_{0}+m \omega\right) t}\left(\frac{c_{m}^{+}}{2} e^{i\left(\omega_{0}+m \omega\right) \xi}+\frac{c_{m}^{-}}{2} e^{-i\left(\omega_{0}+m \omega\right) \xi}\right. \\
& \left.+c_{m}^{+} \sum_{n=0}^{\infty} a_{n}(\xi) e^{\frac{n \pi i}{2}} j_{n}\left(\left(\omega_{0}+m \omega\right) \xi\right)+c_{m}^{-} \sum_{n=0}^{\infty}(-1)^{n} a_{n}(\xi) e^{\frac{n \pi i}{2}} j_{n}\left(\left(\omega_{0}+m \omega\right) \xi\right)\right)
\end{aligned}
$$

and

$$
\begin{aligned}
\mathcal{I}(W(\xi, t))= & \sum_{m=-M}^{M} e^{i\left(\omega_{0}+m \omega\right) t}\left(\frac{c_{m}^{+}}{2} e^{i\left(\omega_{0}+m \omega\right) \xi}-\frac{c_{m}^{-}}{2} e^{-i\left(\omega_{0}+m \omega\right) \xi}\right. \\
& \left.+c_{m}^{+} \sum_{n=0}^{\infty} b_{n}(\xi) e^{\frac{n \pi i}{2}} j_{n}\left(\left(\omega_{0}+m \omega\right) \xi\right)-c_{m}^{-} \sum_{n=0}^{\infty}(-1)^{n} b_{n}(\xi) e^{\frac{n \pi i}{2}} j_{n}\left(\left(\omega_{0}+m \omega\right) \xi\right)\right) .
\end{aligned}
$$

Hence due to (5) and (6) we obtain the following result.

Theorem 2 The unique solution of Problem (1), 15) with the initial data of the form (20) written in terms of coordinates $\xi$ and $t$ has the form

$$
\begin{aligned}
\widetilde{\mathcal{E}}(\xi, t)= & \frac{1}{\sqrt{\widetilde{c}(\xi) \widetilde{\varepsilon}(\xi)}} \sum_{m=-M}^{M} e^{i\left(\omega_{0}+m \omega\right) t}\left(\frac{c_{m}^{+}}{2} e^{i\left(\omega_{0}+m \omega\right) \xi}+\frac{c_{m}^{-}}{2} e^{-i\left(\omega_{0}+m \omega\right) \xi}\right. \\
& \left.+c_{m}^{+} \sum_{n=0}^{\infty} a_{n}(\xi) e^{\frac{n \pi i}{2}} j_{n}\left(\left(\omega_{0}+m \omega\right) \xi\right)+c_{m}^{-} \sum_{n=0}^{\infty}(-1)^{n} a_{n}(\xi) e^{\frac{n \pi i}{2}} j_{n}\left(\left(\omega_{0}+m \omega\right) \xi\right)\right)
\end{aligned}
$$

and

$$
\begin{aligned}
\widetilde{\mathcal{H}}(\xi, t)= & -\frac{i}{\sqrt{\widetilde{c}(\xi) \mu}} \sum_{m=-M}^{M} e^{i\left(\omega_{0}+m \omega\right) t}\left(\frac{c_{m}^{+}}{2} e^{i\left(\omega_{0}+m \omega\right) \xi}-\frac{c_{m}^{-}}{2} e^{-i\left(\omega_{0}+m \omega\right) \xi}\right. \\
& \left.+c_{m}^{+} \sum_{n=0}^{\infty} b_{n}(\xi) e^{\frac{n \pi i}{2}} j_{n}\left(\left(\omega_{0}+m \omega\right) \xi\right)-c_{m}^{-} \sum_{n=0}^{\infty}(-1)^{n} b_{n}(\xi) e^{\frac{n \pi i}{2}} j_{n}\left(\left(\omega_{0}+m \omega\right) \xi\right)\right) .
\end{aligned}
$$


Thus, the solution of Problem (1), (15) with the initial data of the form (20) is obtained in the form of a Neumann series of Bessel functions (see, e.g., [15], 17] and a recent publication on the subject [1] and references therein). Let us discuss an important feature of this solution representation. Namely, for practical computation one needs to consider the truncated series in (23) and (24). Thus, together with the exact solution (23), (24) consider its approximation

$$
\begin{aligned}
\widetilde{\mathcal{E}}_{N}(\xi, t):= & \frac{1}{\sqrt{\widetilde{c}(\xi) \widetilde{\varepsilon}(\xi)}} \sum_{m=-M}^{M} e^{i\left(\omega_{0}+m \omega\right) t}\left(\frac{c_{m}^{+}}{2} e^{i\left(\omega_{0}+m \omega\right) \xi}+\frac{c_{m}^{-}}{2} e^{-i\left(\omega_{0}+m \omega\right) \xi}\right. \\
& \left.+c_{m}^{+} \sum_{n=0}^{N} a_{n}(\xi) e^{\frac{n \pi i}{2}} j_{n}\left(\left(\omega_{0}+m \omega\right) \xi\right)+c_{m}^{-} \sum_{n=0}^{N}(-1)^{n} a_{n}(\xi) e^{\frac{n \pi i}{2}} j_{n}\left(\left(\omega_{0}+m \omega\right) \xi\right)\right)
\end{aligned}
$$

and

$$
\begin{aligned}
\widetilde{\mathcal{H}}_{N}(\xi, t)= & -\frac{i}{\sqrt{\widetilde{c}(\xi) \mu}} \sum_{m=-M}^{M} e^{i\left(\omega_{0}+m \omega\right) t}\left(\frac{c_{m}^{+}}{2} e^{i\left(\omega_{0}+m \omega\right) \xi}-\frac{c_{m}^{-}}{2} e^{-i\left(\omega_{0}+m \omega\right) \xi}\right. \\
& \left.+c_{m}^{+} \sum_{n=0}^{N} b_{n}(\xi) e^{\frac{n \pi i}{2}} j_{n}\left(\left(\omega_{0}+m \omega\right) \xi\right)-c_{m}^{-} \sum_{n=0}^{N}(-1)^{n} b_{n}(\xi) e^{\frac{n \pi i}{2}} j_{n}\left(\left(\omega_{0}+m \omega\right) \xi\right)\right) .
\end{aligned}
$$

It is important to note that for real $\omega_{0}$ and $\omega$ the difference between the exact solution and the approximate one is independent of the largeness of $\omega_{0}$ and $\omega$. More precisely, consider

$$
\left|\widetilde{\mathcal{E}}(\xi, t)-\widetilde{\mathcal{E}}_{N}(\xi, t)\right|=\left|\frac{1}{\sqrt{\widetilde{c}(\xi) \widetilde{\varepsilon}(\xi)}} \sum_{m=-M}^{M} e^{i\left(\omega_{0}+m \omega\right) t}\left(c_{m}^{+} A_{1 ; N}(\omega, \xi)+c_{m}^{-} A_{2 ; N}(\omega, \xi)\right)\right|
$$

where $A_{1 ; N}(\omega, \xi):=\sum_{n=N+1}^{\infty} a_{n}(\xi) e^{\frac{n \pi i}{2}} j_{n}\left(\left(\omega_{0}+m \omega\right) \xi\right)$ and $A_{2 ; N}(\omega, \xi):=\sum_{n=N+1}^{\infty}(-1)^{n} a_{n}(\xi) e^{\frac{n \pi i}{2}}$ $\times j_{n}\left(\left(\omega_{0}+m \omega\right) \xi\right)$. Due to [6, Theorem 4.1] one has $\left|A_{1,2 ; N}(\omega, \xi)\right| \leq \varepsilon_{N}(\xi)$ where $\varepsilon_{N}$ is a positive function which can be made arbitrarily small choosing a sufficiently large $N$. Thus,

$$
\left|\widetilde{\mathcal{E}}(\xi, t)-\widetilde{\mathcal{E}}_{N}(\xi, t)\right| \leq \frac{\varepsilon_{N}(\xi)}{\sqrt{\widetilde{c}(\xi) \widetilde{\varepsilon}(\xi)}} \sum_{m=-M}^{M}\left(\left|c_{m}^{+}\right|+\left|c_{m}^{-}\right|\right)
$$

A similar reasoning is applicable to $(24)$ and (26). One obtains an estimate of the form

$$
\left|\widetilde{\mathcal{H}}(\xi, t)-\widetilde{\mathcal{H}}_{N}(\xi, t)\right| \leq \frac{\varepsilon_{N}(\xi)}{\sqrt{\widetilde{c}(\xi) \mu}} \sum_{m=-M}^{M}\left(\left|c_{m}^{+}\right|+\left|c_{m}^{-}\right|\right) .
$$

The estimates (27) and (28) imply that the approximations (25) and (26) perform equally well for small and for large values of $\omega_{0}$ and $|m \omega|$.

Example $\mathbf{3}$ As an illustration let us consider a situation when the transmutation kernels $\mathbf{K}_{f}$ and $\mathbf{K}_{1 / f}$ admit finite sum representations of the form (9) and (10) and hence the solution (23), (24) admits a closed form expression. As was observed in [8], when

$$
f(\xi)=\frac{1}{(1+\xi)^{2}}
$$

the integral kernels of the transmutation operators $T_{f}$ and $T_{1 / f}$ are given by

$$
\mathbf{K}_{f}(\xi, t)=\frac{(3 t-1)(\xi+1)^{2}-3(t-1)^{2}(t+1)}{4(\xi+1)^{2}} \quad \text { and } \quad \mathbf{K}_{1 / f}(\xi, t)=\frac{3 \xi^{2}+6 \xi+4-3 t^{2}+2 t}{4(\xi+1)} .
$$


They are polynomials with respect to the variable $t$ and hence admit a representation in the form of a finite linear combination of the Legendre polynomials. Simple calculation gives us the following formulas for the coefficients $\left\{a_{n}\right\}$ and $\left\{b_{n}\right\}$ in (9) and (10). We have

$$
\begin{aligned}
& a_{0}(\xi)=-\frac{\xi(\xi+2)}{2(\xi+1)^{2}}, \quad a_{1}(\xi)=\frac{3 \xi^{2}\left(\xi^{2}+5 \xi+5\right)}{10(\xi+1)^{2}}, \quad a_{2}(\xi)=\frac{\xi^{3}}{2(\xi+1)^{2}}, \quad a_{3}(\xi)=-\frac{3 \xi^{4}}{10(\xi+1)^{2}}, \\
& a_{n} \equiv 0, n=4,5, \ldots \text { and } \\
& \quad b_{0}(\xi)=\frac{\xi(\xi+2)}{2}, \quad b_{1}(\xi)=\frac{\xi^{2}}{2(\xi+1)}, \quad b_{2}(\xi)=-\frac{\xi^{3}}{2(\xi+1)}, \quad b_{n} \equiv 0, n=3,4, \ldots
\end{aligned}
$$

As was shown in [5] this example is associated with the following electromagnetic system. Consider system (1) with the permittivity of the form $\varepsilon(x)=(\alpha x+\beta)^{-2+2 \ell}$ where $\ell \neq 0$ and $\alpha, \beta \in \mathbb{R}$ are such that $\alpha x+\beta>0$ on the interval of interest. Then $\xi(x)=\sqrt{\mu} \int_{0}^{x} \sqrt{\varepsilon(s)} d s=\frac{\sqrt{\mu}}{\alpha \ell}\left((\alpha x+\beta)^{\ell}-\beta^{\ell}\right)$. Hence

$$
x=\frac{\beta}{\alpha}\left(\left(1+\frac{\alpha \ell \xi}{\sqrt{\mu} \beta^{\ell}}\right)^{1 / \ell}-1\right), \quad \widetilde{c}(\xi)=\frac{1}{\sqrt{\mu}}\left(\frac{\alpha \ell \xi}{\sqrt{\mu}}+\beta^{\ell}\right)^{(1-\ell) / \ell} \quad \text { and } \quad f(\xi)=\frac{1}{\left(1+\frac{\alpha \ell}{\sqrt{\mu} \beta^{\ell}} \xi\right)^{\frac{1-\ell}{2 \ell}}} .
$$

Thus, take $\ell=1 / 5, \alpha=5, \beta=1$ and $\mu=1$. Then $f$ has the form (29), and solution (23) and (24) of Problem (1), (15) with the initial data of the form (20) admits a finite sum representation obtained from (23) and (24) by substituting the coefficients $\left\{a_{n}\right\}$ and $\left\{b_{n}\right\}$ from this example.

When neither the integrals in 18 can be evaluated in the closed form, nor initial data $\mathcal{E}_{0}$ and $\mathcal{H}_{0}$ can be sufficiently closely approximated by partial sums of Fourier series (20), one faces the problem of efficient numerical evaluation of the integrals in (18) for all required pairs of $t$ and $\xi$. One of the possibilities is to proceed as follows. Denote by $W_{N}(\xi, t)$ the truncated expression (18). We use the explicit representation of the Legendre polynomial, $P_{n}(x)=\sum_{k=0}^{n} l_{k, n} x^{k}$ to obtain that

$$
\begin{aligned}
W_{N}(\xi, t)= & P^{+} W_{0}^{+}(t+\xi)+P^{-} W_{0}^{-}(t-\xi)+\frac{1}{2 \xi} \sum_{n=0}^{N}\left(a_{n}(\xi)+j b_{n}(\xi)\right) \sum_{k=0}^{n} \frac{l_{k, n}}{\xi^{k}} \int_{-\xi}^{\xi} \tau^{k} W_{0}^{+}(t+\tau) \mathrm{d} \tau \\
& +\frac{1}{2 \xi} \sum_{n=0}^{N}\left(a_{n}(\xi)-j b_{n}(\xi)\right) \sum_{k=0}^{n} \frac{l_{k, n}}{\xi^{k}} \int_{-\xi}^{\xi} \tau^{k} W_{0}^{-}(t-\tau) \mathrm{d} \tau .
\end{aligned}
$$

Now we proceed analogously to the derivation of formula (5.2) from [5],

$$
\int_{-\xi}^{\xi} \tau^{k} W_{0}^{+}(t+\tau) \mathrm{d} \tau=\sum_{l=0}^{k}\left(\begin{array}{l}
k \\
l
\end{array}\right)(-1)^{k-l} t^{k-l} \int_{t-\xi}^{t+\xi} z^{l} W_{0}^{+}(z) \mathrm{d} z,
$$

and similarly for the second integral. Hence

$$
\begin{aligned}
W_{N}(\xi, t)= & P^{+} W_{0}^{+}(t+\xi)+\sum_{l=0}^{N}\left(\int_{t-\xi}^{t+\xi} z^{l} W_{0}^{+}(z) \mathrm{d} z\right) \sum_{k=l}^{N} c_{k, N}^{+}(\xi)\left(\begin{array}{c}
k \\
l
\end{array}\right)(-t)^{k-l} \\
& +P^{-} W_{0}^{-}(t-\xi)+\sum_{l=0}^{N}(-1)^{l}\left(\int_{t-\xi}^{t+\xi} z^{l} W_{0}^{-}(z) \mathrm{d} z\right) \sum_{k=l}^{N} c_{k, N}^{-}(\xi)\left(\begin{array}{c}
k \\
l
\end{array}\right) t^{k-l}
\end{aligned}
$$


where

$$
c_{k, N}^{ \pm}(\xi)=\frac{1}{\xi^{k+1}} \sum_{n=k}^{N} \frac{l_{k, n}}{2}\left(a_{n}(\xi) \pm j b_{n}(\xi)\right) .
$$

By such rearrangement we replace the problem of evaluation of the integrals in (18), where for each pair of $t$ and $\xi$ one needs to integrate different functions $P_{n}(\dot{\bar{\xi}}) W_{0}^{ \pm}(t \pm \cdot)$, by the problem of evaluation of the integrals of the same functions $z^{k} W_{0}^{ \pm}(z)$ over different segments. Taking into account that the indefinite integrals $\int_{0}^{x} z^{k} W_{0}^{ \pm}(z) \mathrm{d} z, k=0, \ldots, N$, can be efficiently computed numerically via, e.g., piece-wise polynomial interpolation of the integrands (see, e.g., 3, Section 2.13]), formula (30 may significantly reduce the computational cost. However, one may expect the loss of precision due to multiplication by large coefficients $l_{k, n}\left(\begin{array}{l}k \\ l\end{array}\right)$ and near $\xi=0$ due to division by $\xi^{k+1}$.

Example 4 For the numerical test we consider the problem from Example 6.2 [5]. It consists in the system (1) with the permittivity of the form

$$
\varepsilon(x)=(\alpha x+\beta)^{-2},
$$

where $\alpha$ and $\beta$ are some real numbers, such that $\alpha x+\beta$ does not vanish on the interval of interest. Then $\xi=\sqrt{\mu} \int_{0}^{x} \sqrt{\varepsilon(s)} d s=\frac{\sqrt{\mu}}{\alpha} \log \frac{\alpha x+\beta}{\beta}$. Hence

$$
x=\frac{\beta}{\alpha}\left(e^{\frac{\alpha \xi}{\sqrt{\mu}}}-1\right) \quad \text { and } \quad \widetilde{\varepsilon}(\xi)=\frac{1}{\beta^{2}} e^{-\frac{2 \alpha \xi}{\sqrt{\mu}}}, \quad \widetilde{c}(\xi)=\frac{\beta}{\sqrt{\mu}} e^{\frac{\alpha \xi}{\sqrt{\mu}}}, \quad f(\xi)=e^{-\frac{\alpha \xi}{2 \sqrt{\mu}}} .
$$

In this case the Vekua equation (2) has the form

$$
\partial_{\bar{z}} W+\gamma \bar{W}=0
$$

where the coefficient $\gamma$ is constant, $\gamma=\alpha /(4 \sqrt{\mu})$.

The Vekua equation (32) possesses an exact solution of the form [5]

$$
W(\xi, t)=A e^{i \Omega t}\left(e^{D \xi}+\frac{D+C}{D-C} e^{-D \xi}+\frac{2 i j \Omega}{D-C} \sinh D \xi\right)
$$

here $A$ and $\Omega$ are arbitrary constants, $C=\frac{\alpha}{2 \sqrt{\mu}}, D=i \sqrt{\Omega^{2}-C^{2}}$.

Hence (returning to the variable $x$ )

$$
\mathcal{E}(x, t)=A \sqrt[4]{\mu} \sqrt{\alpha x+\beta} e^{i \Omega t}\left(\left(\frac{\alpha x+\beta}{\beta}\right)^{\frac{D \sqrt{\mu}}{\alpha}}+\frac{D+C}{D-C}\left(\frac{\alpha x+\beta}{\beta}\right)^{-\frac{D \sqrt{\mu}}{\alpha}}\right)
$$

and

$$
\mathcal{H}(x, t)=\frac{A}{D-C} \frac{\Omega e^{i \Omega t}}{\sqrt[4]{\mu} \sqrt{\alpha x+\beta}}\left(\left(\frac{\alpha x+\beta}{\beta}\right)^{\frac{D \sqrt{\mu}}{\alpha}}-\left(\frac{\alpha x+\beta}{\beta}\right)^{-\frac{D \sqrt{\mu}}{\alpha}}\right)
$$

satisfy the Maxwell system (1) with the permittivity (31) and the initial conditions

$$
\mathcal{E}(0, t)=\frac{2 A D}{D-C} \sqrt[4]{\mu} \sqrt{\beta} e^{i \Omega t} \quad \text { and } \quad \mathcal{H}(0, t)=0 .
$$

For the numerical calculation we considered an interval $[0,6]$ for both $x$ and $t$ and took $\alpha=2$, $\beta=1, \mu=1$. For the initial condition we took the sum of four terms, each of the form (33) 
having $\Omega_{1}=-\Omega_{2}=C+1, \Omega_{3}=-\Omega_{4}=C+2$. Since the expression (33) for $\xi=0$ reduces to $W(0, t)=\frac{2 A D}{D-C} e^{i \Omega t}$, we took $A_{i}=\frac{D_{i}-C}{D_{i}}, i=1, \ldots, 4$ and obtained the initial data $W_{0}^{ \pm}(t)=$ $4 \cos (C+1) t+4 \cos (C+2) t$.

We compared solutions computed by (18) and (30). All calculations were performed using Matlab 2017 in the machine precision. The exact expressions were used only for the function $\varepsilon(x)$ and its derivatives, all other functions involved were computed numerically.

The permittivity $\varepsilon(x)$ was approximated on uniform mesh of 5001 points. The new variable $\xi$ was obtained by the modified 6 point Newton-Cottes integration formula, see [6] for details. The same integration formula was used for calculation of the coefficients $a_{n}$ and $b_{n}$ and the integrals in (18). Note that the integration with respect to the variable $\xi$ requires integration over a nonuniform mesh, however such inconvenience can be easily avoided observing that $\int_{0}^{\xi(x)} \widetilde{g}(\xi) d \xi=$ $\int_{0}^{x} g(s) \xi^{\prime}(s) d s=\int_{0}^{x} g(s) \sqrt{\mu \varepsilon(s)} d s$ for any function $g(x)=\widetilde{g}(\xi(x))$. To evaluate the indefinite integrals $\int z^{k} W_{0}^{ \pm}(z) d z, k=0, \ldots, N$ we approximated the integrands as splines and used the function fnint from Matlab. The main reason for such choice is that the set of values taken by $t-\xi$ and $t+\xi$ may be rather large and need not to be uniformly spaced and so some kind of interpolation is necessary, we opted for splines.

On Figure 1 we show the permittivity (on the left) and the initial data $W_{0}^{ \pm}(t)$ (on the right).
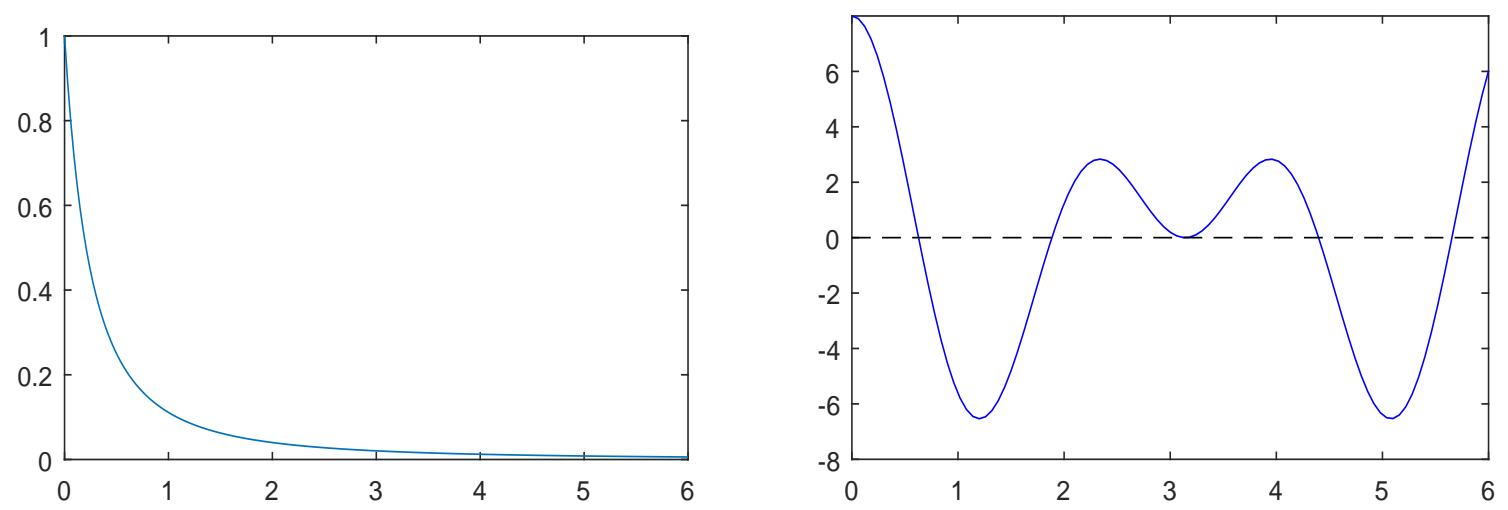

Figure 1: Graphs of the permittivity $\varepsilon(x)$ (on the left) and the initial data $W_{0}^{ \pm}(t)$ (on the right, real part in solid blue line, imaginary part in dashed black line) from Example 4.

On Figure 2 we show the graphs of the solutions $\mathcal{E}(x, t)$ (on the left) and $\mathcal{H}(x, t)$ (on the right, imaginary part). Note that for the chosen initial data the values of $\mathcal{E}(x, t)$ are real (so that $\mathcal{E}$ coincides with the component $E_{2}$ ), while the values of $\mathcal{H}(x, t)$ are purely imaginary (so that the imaginary part of $\mathcal{H}$ coincides with the component $H_{3}$ )

The developed program found the optimal value of $N$ for the approximation of the transmutation operators to be $N=13$ (see [6] for related details). On Figure 3 we show the graphs of the absolute errors of the computed $\mathcal{E}(x, t)$ and $\mathcal{H}(x, t)$ using directly formula (18). One can appreciate excellent accuracy, however the computation time of the solutions on the mesh of $201 \times 101$ points $(x, t)$ was about 4 minutes.

The computation time required by formula (30) was only 4 seconds, and most of this time was spent for the construction of splines. On Figure 4 (top graphs) we show the graphs of the absolute errors of the computed $\mathcal{E}(x, t)$ and $\mathcal{H}(x, t)$. As one can see, the error is close to machine precision limit away from $x=0$, however rapidly increasing as $x$ approaches 0 due to division by large powers of $\xi$ in the coefficients $c_{k, N}^{ \pm}$.

The situation can be improved by taking smaller value of $N$ for this region. For example, for $N=6$ the maximum absolute error reduces to $7 \cdot 10^{-8}$, however the errors for values of $x$ distant 

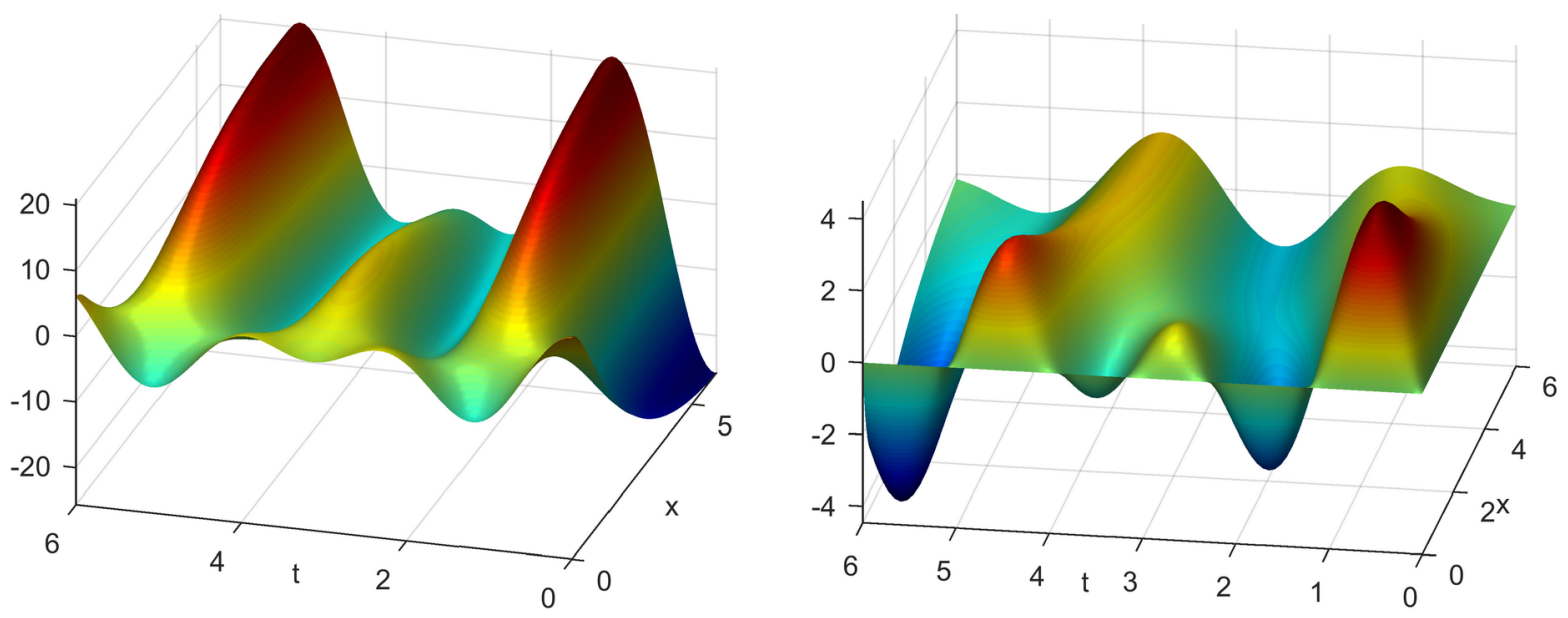

Figure 2: Graphs of the exact solutions $\mathcal{E}(x, t)$ (on the left) and $\mathcal{H}(x, t)$ (on the right, imaginary part) from Example 4.
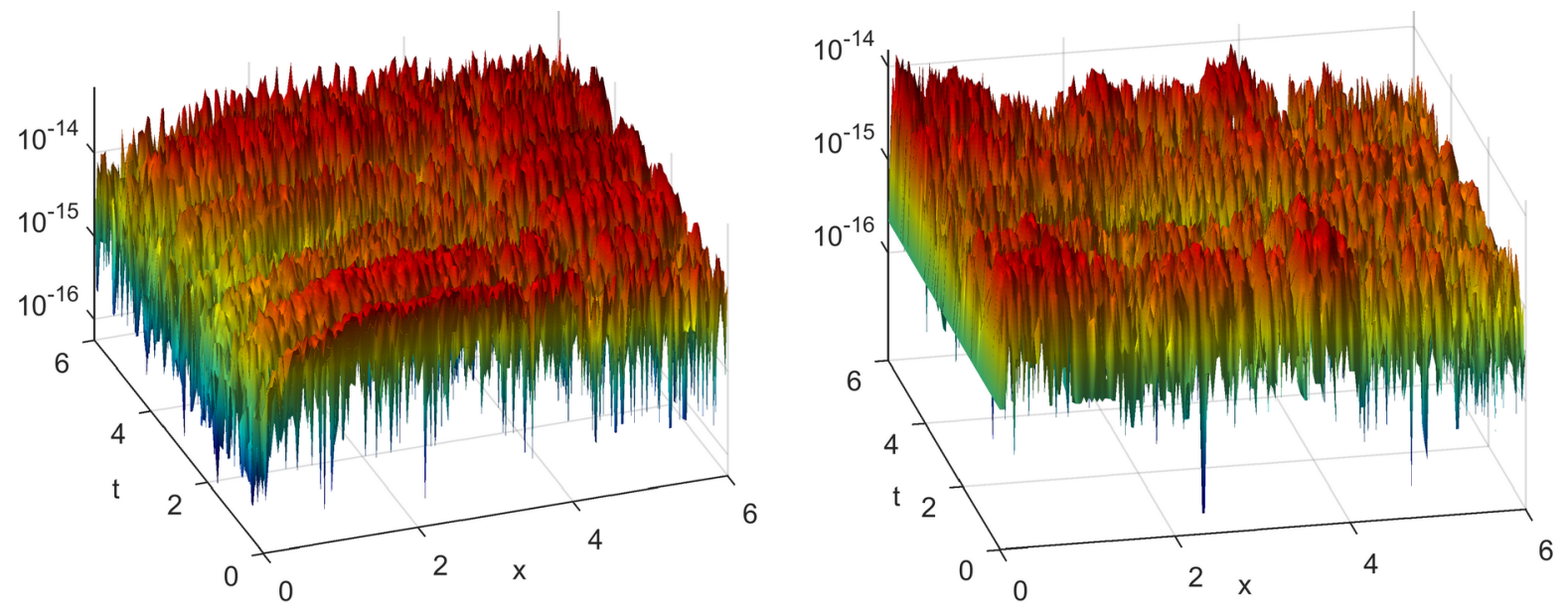

Figure 3: Graphs of the absolute errors of $\mathcal{E}(x, t)$ (on the left) and $\mathcal{H}(x, t)$ (on the right) computed using formula (18) truncated to $N=13$.

from 0 growth. See Figure 4 (bottom row).

\section{References}

[1] A. Baricz, D. Jankov and T. K. Pogány, Neumann series of Bessel functions, Integral Transforms Spec. Funct. 23 (2012), no. 7, 529-538.

[2] H. Campos, V. V. Kravchenko, L. M. Méndez, Complete families of solutions for the Dirac equation: an application of bicomplex pseudoanalytic function theory and transmutation operators, Adv. Appl. Clifford Algebr. 22 (2012), issue 3, 577-594.

[3] P. J. Davis and P. Rabinowitz, Methods of numerical integration. Second edition, New York: Dover Publications, 2007. 


$$
N=13
$$
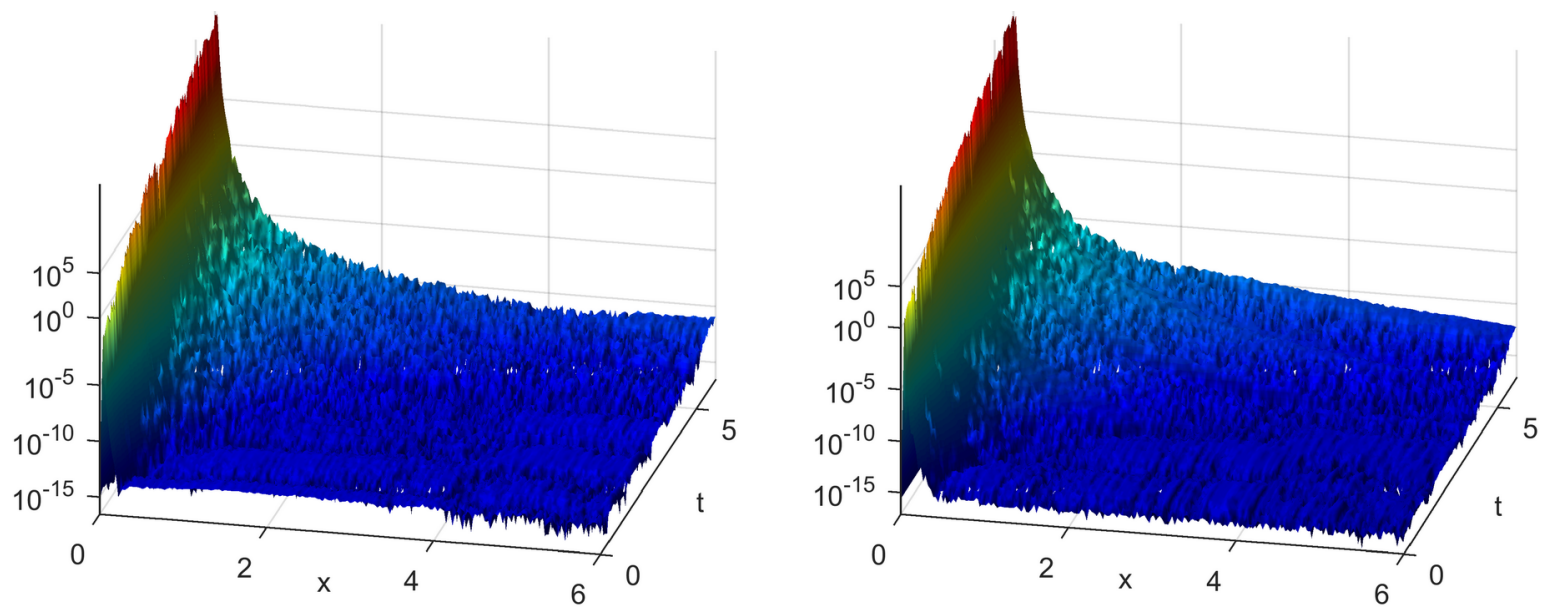

$N=6$
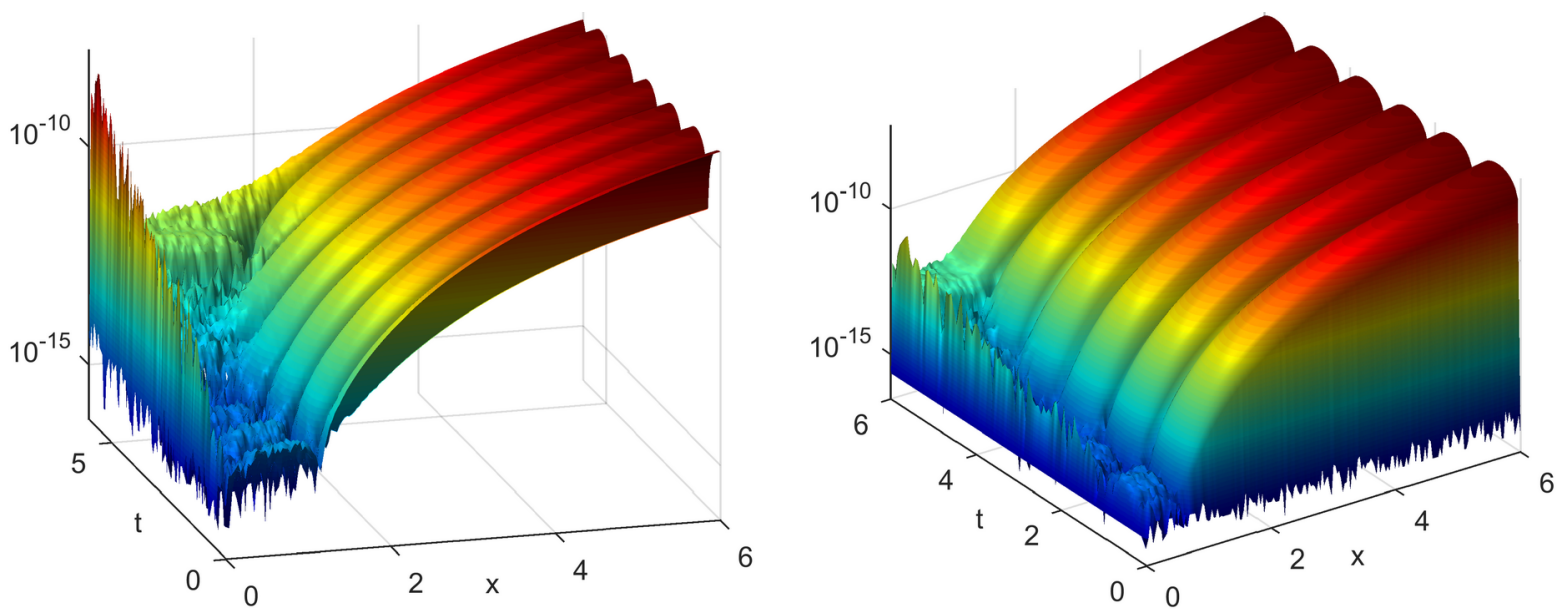

Figure 4: Graphs of the absolute errors of $\mathcal{E}(x, t)$ (on the left) and $\mathcal{H}(x, t)$ (on the right) computed using formula (30). Top graphs: using $N=13$, bottom graphs: using $N=6$.

[4] V. V. Katrakhov, S. M. Sitnik, The transmutation method and boundary value problems for singular elliptic equations. Contemporary Mathematics. Fundamental Directions. Vol. 64, Iss. 2, p. 211-426. (in Russian).

[5] K. V. Khmelnytskaya, V. V. Kravchenko and S. M. Torba, Modulated electromagnetic fields in inhomogeneous media, hyperbolic pseudoanalytic functions and transmutations, J. Math. Phys. 57 (2016), 051503 (10pp.).

[6] V. V. Kravchenko, L. J. Navarro, S. M. Torba, Representation of solutions to the onedimensional Schrödinger equation in terms of Neumann series of Bessel functions, Appl. Math. Comput. 314 (2017), 173-192.

[7] V. V. Kravchenko, M. P. Ramirez, On Bers generating functions for first order systems of mathematical physics, Adv. Appl. Clifford Algebr. 21 (2011), issue 3, 547-559.

[8] V. V. Kravchenko and S. M. Torba, Transmutations for Darboux transformed operators with applications, J. Phys. A: Math. Theor., 45 (2012), \# 075201 (21 pp.).

[9] V. V. Kravchenko and S. M. Torba, Construction of transmutation operators and hyperbolic pseudoanalytic functions, Complex Anal. Oper. Theory 9 (2015), 379-429. 
[10] M. A. Lavrentyev and B. V. Shabat, Hydrodynamics problems and their mathematical models. Nauka, Moscow, 1977 (in Russian).

[11] A. E. Motter and M. A. F. Rosa, Hyperbolic calculus, Adv. Appl. Clifford Algebr. 8 (1998) 109-128.

[12] L. A. Ostrovsky, A. I. Potapov, Introduction into the theory of modulated waves, Fizmatlit, Moscow, 2003 (in Russian), the revised and extended translation of Modulated Waves: Theory and Applications, Johns Hopkins University Press, 2002.

[13] A. P. Prudnikov, Yu. A. Brychkov and O. I. Marichev, Integrals and series. Vol. 2. Special functions. Gordon \& Breach Science Publishers, New York, 1986. 750 pp.

[14] S. M. Sitnik, E. L. Shishkina Method of transmutations for differential equations with Bessel operators. 2019, Moscow, Fizmatlit, 224 p. (in Russian).

[15] G. N. Watson, A Treatise on the theory of Bessel functions, 2nd ed., reprinted, Cambridge: Cambridge University Press, 1996, vi+804 pp.

[16] G. C. Wen, Linear and Quasilinear Complex Equations of Hyperbolic and Mixed Type, Taylor \& Francis London, 2003.

[17] J. E. Wilkins, Neumann series of Bessel functions, Trans. Amer. Math. Soc. 64 (1948), 359385. 